\title{
A CONVOLUTION BACK PROJECTION ALGORITHM FOR LOCAL TOMOGRAPHY
}

\author{
CHALLA S. SASTRY ${ }^{1}$ and P. C. DAS ${ }^{2}$
}

(Received 28 July, 2003; revised 15 February, 2004)

\begin{abstract}
The present work deals with the problem of recovering a local image from localised projections using the concept of approximation identity. It is based on the observation that the Hilbert transform of an approximation identity taken from a certain class of compactly supported functions with sufficiently many zero moments has no significant spread of support. The associated algorithm uses data pertaining to the local region along with a small amount of data from its vicinity. The main features of the algorithm are simplicity and similarity with standard filtered back projection (FBP) along with the economic use of data.
\end{abstract}

\section{Introduction}

The problem of recovering a local image from localised projections in X-ray tomography has acquired importance so that the radiation dosage as well as the time of exposure can be reduced. The problems relating to uniqueness, well-posedness etc. associated with local inversion of the Radon transform are well known [14]. Recently, wavelet-based methods have proved quite promising for the purpose of Region-OfInterest (ROI) tomography [7,15]. One method based on angular harmonics [18] uses projections, exponentially sampled in radial directions, whereby finer sampling is achieved in the ROI and coarser sampling outside it. Nonetheless, this is not a local reconstruction in the true sense, as full length projections are required along certain directions and the computations involve interpolations. Several other wavelet-based methods are also available $[5,7,15]$, which use a reasonably small amount of neighbourhood data in addition to ROI data. The amount of additional data depends on the

\footnotetext{
'Arificial Intelligence Lab, Department of Computer and Information Sciences, University of Hyderabad, Hyderabad 500046, India; e-mail: s.challa@lycos.com.

${ }^{2}$ Department of Mathematics, Indian Institute of Technology, Kanpur 208016, India; e-mail: pcdas@iitk.ac.jn.

(C) Australian Mathematical Society 2005, Serial-fee code 1446-1811/05
} 
choice of wavelets used.

The aim of the present work is to propose a filtered back projection (FBP)-type formula for ROI reconstruction using the concept of an approximation identity. The work of Rieder et al. $[16,17]$ is conceptually related to the present work although the purpose and details are different. The proposed algorithm, using an approximation identity taken from a class of compactly supported functions, has the following main advantages: (i) it does not use exponential radial sampling and is therefore simple to implement; (ii) it allows uniform exposure at all angles as in [15]; and (iii) it is analogous to the standard FBP algorithm and hence can use several readymade well-developed procedures for implementations [2].

The present work is organised into the following six sections. In Section 2, we present some standard notation, the basic reconstruction technique and make some observations about the nonlocality of the Hilbert transform. In Section 3, we consider the local reconstruction technique using the concept of an approximation identity. Since the idea is simple, we have tried to derive the representation formula directly without referring to any other technical notation. Section 4 deals with the error estimate depending upon the parameter of the approximation identity and the zero moments satisfied by the corresponding function. In Section 5 , we construct some examples of the approximation identity. In Section 6, we study the error arising from the use of localised data from interior regions. Section 7 deals with a comparison of the results of this paper with those of Faridani et al. [8-10], Rieder et al. $[16,17]$, A. K. Louis [11] and other wavelet-based methods $[5,7,15]$. In the last section, we consider some simulation results indicating how to use the right parameters.

\section{Preliminaries and the reconstruction techniques}

Before developing the reconstruction algorithm, we state some basic definitions and provide information regarding the Hilbert transform, to be used subsequently.

2.1. Basic reconstruction formula Let $L^{2}\left(\mathbb{R}^{2}\right)$ and $H^{\alpha}\left(\mathbb{R}^{2}\right)$ denote respectively the space of all square integrable functions and the Sobolev space of order $\alpha$ on $\mathbb{R}^{2}$. Then the norm of the function $f \in H^{\alpha}\left(\mathbb{R}^{2}\right)[1]$ is given by

$$
\|f\|_{H^{a}\left(\mathbb{R}^{2}\right)}=\left(\int_{\mathbb{R}^{2}}|\hat{f}(\omega)|^{2}\left(1+\|\omega\|^{2}\right)^{\alpha} d \omega\right)^{1 / 2}<\infty .
$$

The Fourier transform of $f$ is defined by

$$
\hat{f}(\omega)=\int_{\mathbb{R}^{n}} f(x) e^{-2 \pi \imath(x, \omega)} d x
$$


Here $\langle x, \omega\rangle$ stands for the inner product of $x$ and $\omega$ in $\mathbb{R}^{2}$. The Radon transform $\left(R_{\theta} f\right)$ of a sufficiently regular function $f$ on $\mathbb{R}^{2}$ is given for $\theta \in[0,2 \pi)$ and $t \in(-\infty, \infty)$ by the formula

$$
\left(R_{\theta} f\right)(t)=\int_{\mathbb{R}} f\left(t u_{\theta}+s v_{\theta}\right) d s,
$$

where $u_{\theta}=(\cos \theta, \sin \theta)$ and $v_{\theta}=u_{\theta+\pi / 2}$.

The basic objective in tomography is to reconstruct from the projection data $R_{\theta} f$ the density function $f: \mathbb{R}^{2} \rightarrow \mathbb{R}$ having support in a disc of radius $R$ centred at the origin in $\mathbb{R}^{2}$. The basic reconstruction technique is based on the following result called the slice theorem [14]:

$$
\widehat{\left(R_{\theta} f\right)}(\omega)=\hat{f}\left(\omega u_{\theta}\right),
$$

expressing the Fourier transform of $f$ in terms of the Fourier transforms of $\left(R_{\theta} f\right)$ for $0 \leqslant \theta<2 \pi$.

Using the Fourier inversion formula in polar form and the slice theorem, the function $f$ is reconstructed from the Radon projection data $\left(R_{\theta} f\right)$ by

$$
f(x)=\int_{0}^{\pi} \int_{\mathbb{R}} \widehat{\left(R_{\theta} f\right)}(\omega) e^{i 2 \pi \omega\left(x, u_{\theta}\right)}|\omega| d \omega d \theta .
$$

Equation (2.5) is called the back projection formula.

2.2. Effect of nonlocality In (2.5) the Fourier transform of $R_{\theta} f$ is multiplied by $|\omega|$. In the spatial domain this product can be written in terms of the Hilbert transform as follows:

$$
\operatorname{IFT}\left(\mid \cdot \widehat{\left(R_{\theta} f\right)}\right)(t)=H \partial\left(R_{\theta} f\right)(t):=\left(\Lambda_{\theta} f\right)(t) .
$$

In fact, for any sufficiently regular univariable function $g$ we have

$$
H \partial g(\eta)=(\Lambda g)(\eta)
$$

It can be seen that

$$
\widehat{(H g)}(\omega)=\frac{\operatorname{sgn}(\omega)}{i} \hat{g}(\omega) .
$$

Hence the Fourier transform of a function with nonzero average value has discontinuity at the origin. The presence of discontinuity at the origin in the frequency domain has the effect of spreading the support of the Hilbert transform of a compactly supported function [14]. However, the essential support of the filtered function does not spread if the function has a sufficient number of zero moments $[14,15]$. Next we come to the main reconstruction formula of the present paper. 


\section{CBP based on an approximation identity}

We start with the following simple lemma on an approximation identity (see for example [3, Theorem 2.8]).

LEMMA 3.1. Let $\phi$ be a compactly supported continuous function such that

$$
\int_{\mathbb{R}} \phi(t) d t=1
$$

and let $g \in L^{1}(\mathbb{R})$, then at any point $x$ of continuity of $g$

$$
\lim _{j \rightarrow \infty}\left(g \star \phi_{j}\right)(x)=g(x), \text { where } \phi_{j}=2^{\jmath} \phi\left(2^{\jmath}\right) .
$$

Now we recollect that $\Lambda_{\theta} f$ stands for $H \partial\left(R_{\theta} f\right)$. In view of the above lemma, at the points of continuity ' $t$ ' of $\left(\Lambda_{\theta} f\right)$

$$
\lim _{j \rightarrow \infty}\left[\left(\Lambda_{\theta} f\right) \star \phi_{J}\right](t)=\left(\Lambda_{\theta} f\right)(t)
$$

Using the definitions given in (2.6) and (2.7), we obtain the following important identity, which is basic to our later considerations.

$$
\left[\left(\Lambda_{\theta} f\right) \star \phi_{j}\right]=\operatorname{IFT}\left[\widehat{\left(R_{\theta} f\right)}|\cdot| \hat{\phi}_{j}\right]=\left(R_{\theta} f\right) \star \operatorname{IFT}\left(|\cdot| \hat{\phi}_{j}\right)=\left(R_{\theta} f\right) \star\left(\Lambda \phi_{j}\right) .
$$

In the above, IFT stands for the inverse Fourier transform operation. From the standard back projection formula and (3.1), we have the following approximate reconstruction formula for sufficiently large $J$ :

$$
\begin{aligned}
f(x) & =\int_{0}^{\pi}\left(\Lambda_{\theta} f\right)\left(\left\langle x, u_{\theta}\right\rangle\right) d \theta \approx \int_{0}^{\pi}\left[\left(\Lambda_{\theta} f\right) \star \phi_{J}\right]\left(\left\langle x, u_{\theta}\right\rangle\right) d \theta \\
& =\int_{0}^{\pi}\left[\left(R_{\theta} f\right) \star\left(\Lambda \phi_{J}\right)\right]\left(\left(x, u_{\theta}\right\rangle\right) d \theta \\
& =2^{J} \int_{0}^{\pi}\left[\left(R_{\theta} f\right) \star(\Lambda \phi)_{J}\right]\left(\left\langle x, u_{\theta}\right\rangle\right) d \theta=f_{\phi, J}(x) \quad \text { (say). }
\end{aligned}
$$

Here we have used the easily verifiable relations $\left(\Lambda \phi_{J}\right)(s)=2^{2 J}(\Lambda \phi)\left(2^{s} s\right)$ and $g_{J}(s)=2^{J} g\left(2^{J} s\right)$. We note that $f_{\eta, j}(x)$ stands for

$$
f_{\eta, j}(x)=2^{j} \int_{0}^{\pi}\left[\left(R_{\theta} f\right) \star(\Lambda \eta)_{l}\right]\left(\left(x, u_{\theta}\right)\right) d \theta .
$$


REMARK 1. Since the function $\phi$ is a mollifier, its scale parameter $J$ acts as a regularisation parameter and the method in (3.2) is a regularisation method. When the function $\phi$ has no significant spread of support after ramp filtering, the above formula gives a local reconstruction procedure. Although taking larger values for $J$ reduces the excess (outside the ROI) data commensurately, it also results in spreading the support of the ramp function and the consequent magnification of noise due to the ramp function. On the other hand taking smaller values for $J$ leads to higher approximation errors in (3.2) in the act of approximating $\left(\Lambda_{\theta} f\right)$ by $\left(\Lambda_{\theta} f\right) \star \phi_{J}$. In view of this, a balanced value of $J$ has to be used. We discuss this point while analysing simulation results.

REMARK 2. Since $\hat{\phi}(0)=1$, the action of the approximation identity on $f$ may result in the low-bandpass-filtered version of $f$. Now, suppose that $\psi$ is a compactly supported, smooth function such that $\hat{\psi}(0)=0$. Then the function $\tilde{\phi}=\phi+\lambda \psi$ still acts as an approximation identity and hence $\phi$ in (3.2) can be replaced by $\tilde{\phi}$. The parameter $\lambda$ may be used as a control parameter for the enhancement of the image. Inserting $\tilde{\phi}$ in place of $\phi$ in (3.2) and using the linearity of the convolution operation we get

$$
\begin{aligned}
f_{\tilde{\phi}, J}(x)= & 2^{J} \int_{0}^{\pi}\left[\left(R_{\theta} f\right) \star(\Lambda \phi)_{J}\right]\left(\left(x, u_{\theta}\right)\right) d \theta \\
& +\lambda 2^{J} \int_{0}^{\pi}\left[\left(R_{\theta} f\right) \star(\Lambda \psi)_{J}\right]\left(\left(x, u_{\theta}\right\rangle\right) d \theta \\
= & f_{\phi, J}(x)+\lambda f_{\psi, J}(x) .
\end{aligned}
$$

The second part in (3.4) may be regarded as a high-bandpass-filtered version of $f$. So far the choices of $\psi$ and $\phi$ have been independent. In fact, it is possible to choose several $\psi$ 's like $\psi_{1}, \psi_{2}, \ldots, \psi_{p}$ and get a more generalised expression for (3.4).

When the function $\psi$ considered is oscillatory and orthogonal to $\phi$, that is, $\langle\phi, \psi\rangle=0$, the function $f_{\psi, J}$ captures information that is complementary to that captured by the $\phi$-part in the sense that the oscillations are better captured by $\psi$. There is, however, no heuristic rule for the determination of the parameter $\lambda$.

In the next section we present an estimate of the error arising due to a finite choice of $J$.

\section{Error estimate}

As noted above, finding proper values of $J$ is an important aspect to be dealt with. One has to balance the conflicting effects on the approximation error of the small value 
of $J$ and the spread of frequency support and consequent dominance of high frequency noise for large values of $J$. In this section we provide an estimate which helps in some measure to choose the proper values for $J$. We assume that the function $\phi$ besides satisfying the aforementioned properties has support in $[-a, a]$ and possesses $M$ zero moments, that is,

$$
\int_{\mathbb{R}} s^{i} \phi(s) d s=0, \quad i=1,2, \ldots, M .
$$

For $t \in[-R, R]$, let $E(t)$ be the error arising due to the approximation of $\left(\Lambda_{\theta} f\right)(t)$ by $\left[\left(\Lambda_{\theta} f\right) \star \phi_{J}\right](t)$. Then taking a Taylor series expansion up to $N$ terms (where $N-1 \leq M)$ and using the zero moment property of $\phi$ we have

$$
\begin{aligned}
E(t) & =\left[\left(\Lambda_{\theta} f\right) \star \phi_{J}\right](t)-\left(\Lambda_{\theta} f\right)(t) \\
& =\int_{\mathbb{R}}\left[\left(\Lambda_{\theta} f\right)(t-s)-\left(\Lambda_{\theta} f\right)(t)\right] \phi_{J}(s) d s \quad\left[\text { using } \int_{\mathbb{R}} \phi(s) d s=1\right] \\
& =\int_{\mathbb{R}}\left[\sum_{l=0}^{N-1} \frac{(-s)^{l}}{l !} \partial^{l}\left(\Lambda_{\theta} f\right)(t)+\frac{(-s)^{N}}{N !} \partial^{N}\left(\Lambda_{\theta} f\right)\left(c_{s, t}\right)-\left(\Lambda_{\theta} f\right)(t)\right] \phi_{J}(s) d s \\
& =\int_{\mathbb{R}} \frac{\left(-2^{-J} s\right)^{N}}{N !} \partial^{N}\left(\Lambda_{\theta} f\right)\left(c_{s, t}^{J}\right) \phi(s) d s=\int_{-a}^{a} \frac{\left(-2^{-J} s\right)^{N}}{N !} \partial^{N}\left(\Lambda_{\theta} f\right)\left(c_{s, t}^{J}\right) \phi(s) d s,
\end{aligned}
$$

where we have used that $\operatorname{supp} \phi=[-a, a]$ and $N-1 \leq M$. Taking the modulus on both sides of the above equation, we have

$$
\begin{aligned}
|E(t)| & \leq \frac{2^{-J N}}{N !} \int_{-a}^{a}\left|\partial^{N}\left(\Lambda_{\theta} f\right)\left(c_{s, l}^{J}\right)\right|\left|s^{N} \phi(s)\right| d s \\
& \leq \frac{2^{-J N}}{N !}\left\|(\cdot)^{N} \phi\right\|_{L^{\prime}(\mathbb{R})} \sup _{1-R-a, R+a]}\left|\partial^{N}\left(\Lambda_{\theta} f\right)\right| .
\end{aligned}
$$

Since $\left(\Lambda_{\theta} f\right)(s)=\int_{\mathbb{R}} \widehat{\left(R_{\theta} f\right)}(t)|t| e^{2 \pi i t s} d t$, we have

$$
\partial^{N}\left(\Lambda_{\theta} f\right)(s)=\int_{\mathbb{R}} \widehat{\left(R_{\theta} f\right)}(t)|t|(2 \pi i t)^{N} e^{2 \pi i s t} d t
$$

and hence

$$
\left|\partial^{N}\left(\Lambda_{\theta} f\right)(s)\right| \leq(2 \pi)^{N} \int_{\mathbb{R}}\left|\widehat{\left(R_{\theta} f\right)}(t)\right||t|^{N+1} d t
$$

Now, using (4.1), (4.2) and (2.4) we get

$$
\begin{aligned}
\int_{0}^{\pi}\left|E\left(\left\langle x, u_{\theta}\right\rangle\right)\right| d \theta & \leq \frac{2^{-J N}}{N !}\left\|(\cdot)^{N} \phi\right\|_{L^{\prime}(\mathbb{R})}(2 \pi)^{N} \int_{0}^{\pi} \int_{\mathbb{R}}\left|\widehat{\left(R_{\theta} f\right)}(t)\right||t|^{N+1} d t d \theta \\
& =\frac{2^{-J N}}{N !}\left\|(\cdot)^{N} \phi\right\|_{L^{\prime}(\mathbb{R})}(2 \pi)^{N} \int_{\mathbb{R}^{2}}|\hat{f}(\xi)|\|\xi\|^{N} d \xi .
\end{aligned}
$$


Now, estimating the last integral in the last line of the above inequality and using (2.1), we get

$$
\begin{aligned}
\int_{\mathbb{R}^{2}}|\hat{f}(\xi)|\|\xi\|^{N} d \xi & \leq \int_{\mathbb{R}^{2}}|\hat{f}(\xi)|\left(1+\|\xi\|^{2}\right)^{N / 2} d \xi \\
& \leq \int_{\mathbb{R}^{2}} \frac{|\hat{f}(\xi)|\left(1+\|\xi\|^{2}\right)^{(N+1) / 2+\epsilon}}{\left(1+\|\xi\|^{2}\right)^{1 / 2+\epsilon}} d \xi \\
& \leq\left(\int_{\mathbb{R}^{2}}|\hat{f}(\xi)|^{2}\left(1+\|\xi\|^{2}\right)^{N+1+2 \epsilon} d \xi\right)^{1 / 2}\left(\int_{\mathbb{R}^{2}} \frac{d \xi}{\left(1+\|\xi\|^{2}\right)^{1+2 \epsilon}}\right)^{1 / 2} \\
& =\sqrt{\frac{\pi}{2 \epsilon}}\|f\|_{H^{N+1+2 e}\left(\mathbb{R}^{2}\right)} .
\end{aligned}
$$

In the above inequality $\epsilon$ is any positive quantity. In particular, when we take $\epsilon=1 / 2$, we get

Thus we conclude that

$$
\begin{aligned}
& \left\|f-f_{\phi, J}\right\|_{L^{\infty}}=\underset{x \in \mathbb{R}^{2}}{\operatorname{ess} \sup }\left|\int_{0}^{\pi} E\left(\left\langle x, u_{\theta}\right\rangle\right) d \theta\right| \\
& \leq \frac{2^{-J N}}{N !}\left\|(\cdot)^{N} \phi\right\|_{L^{1}(\mathbb{R})}(2 \pi)^{N} \sqrt{\pi}\|f\|_{H^{N+2}\left(\mathbb{R}^{2}\right)} .
\end{aligned}
$$

$$
\left\|f-f_{\phi, J}\right\|_{L^{\infty}}=O\left(\frac{2^{-J N}}{N !}\right) \text { provided } f \in H^{N+2}\left(\mathbb{R}^{2}\right) .
$$

REMARK 3. Note that the function $f_{\phi, J}$ given in (3.2) can be written as

$$
f \approx f_{\phi, J}=f \star \Phi^{J}=\Re^{\sharp}\left(\Re f \star \Lambda \phi_{J}\right),
$$

where $\mathfrak{R}^{\#}$ is the adjoint of the Radon transform operator $\Re$ and $\Phi^{J}$ stands for $\mathfrak{R}^{\#} \Lambda \phi_{J}$. It may be observed that $\widehat{\Phi^{J}}(\xi)=\hat{\phi}_{J}(\|\xi\|)$ and hence $\Phi^{\prime}$ satisfies $\int_{\mathbb{R}^{2}} \Phi^{J}(x) d x=1$ and zero moments as $\phi$. Consequently, estimates of the type

$$
\left\|f-f \star e_{\gamma}^{n}\right\|_{H^{\prime}\left(\mathbb{R}^{2}\right)} \leq C \gamma^{3}\|f\|_{H^{r+3}\left(\mathbb{R}^{2}\right)}
$$

hold as shown in [16, Example 3.4]. In (4.5), $e_{\gamma}^{n}$ is a radial mollifier defined as $\left(1 / \gamma^{2}\right) e^{n}(1 / \gamma)$ for $\gamma>0$, where the function $e^{n}$ has zero moments as shown in [16, Example 3.4].

The estimate proved in (4.5), like the estimate (4.3) proved in this section, shows faster convergence provided $f$ has a sufficient degree of smoothness. The $L^{\infty}$ error present in (4.3) captures the localised error better than any other error estimate. For example, any slight shift in the pixel values of the reconstructed image is better identified by the $L^{\infty}$ error than by the error involving a Sobolev norm in (4.5). 
Generally in applications, we reconstruct pictures that are smooth except for some jumps and such simple pictures relate to density functions in $H^{1 / 2}$ [14], the Sobolev space of order $1 / 2$. However, in view of the above estimates to achieve faster convergence, one requires a higher degree of smoothness for $f$ and sufficiently many zero moments of $\phi$. From (4.3), it is evident that higher order smoothness for $f$ (that is, larger values of $N$ ) can compensate the lower values of $J$, in order to achieve a similar error estimate. Since the function $\phi$ has higher order zero moments and is sufficiently smooth, it decays faster in the frequency domain as well, and as a result $\phi$ acts as a low pass filter. Consequently, a smaller $J$ may be helpful in interpolating the data function with fewer samples.

An estimate of type (4.3) holds even when we use $\tilde{\phi}=\phi+\lambda \psi$ in (3.2) with $\phi, \psi$ satisfying the following zero moment conditions: $\int_{\mathbb{R}} s^{i} \phi(s) d s=\delta_{i, 0}$ and $\int_{\mathbb{R}} s^{i} \psi(s) d s=0, i=0,1, \ldots, M$.

\section{Examples of $\phi$}

It is well known (see [6]) that coiflets and their corresponding scaling functions satisfy zero moment properties. It is also known [14] that the Hilbert transforms of sufficiently regular functions possessing zero moments of sufficient order do not show spread in support. Similarly if wavelet functions of sufficient regularity satisfy the zero moment property, then the supports of $H \psi$ and $\psi$ are not essentially different. However, the scaling functions have unit mean value and hence do not satisfy this property in general. Nevertheless, for certain classes of scaling functions, the function $\Lambda \phi$ is approximately finitely supported (in the sense that $\Lambda \phi$ takes negligible values outside some finite interval) as documented in [15]. The interval over which $\Lambda \phi$ takes significant values is called the essential support of $\Lambda \phi$. In computation the essential support is normally taken based on some error criterion, which is discussed in later sections. The coiflet scaling functions satisfy the stated desirable properties.

Although the coiflet scaling function is a suitable candidate for $\phi$, it lacks closed form. If one wants to use $\phi$ with a closed form, one can construct it in the following way. Since the procedure given in the present work is not a wavelet-based method and one can use any $\phi, \psi$ satisfying the conditions assumed for them, it should be remarked that the use of the $\psi$-part is not a requirement of the procedure. In the following, we present the construction of a $\phi$ having closed form.

We start with symmetric, compactly supported and sufficiently smooth functions $S_{i}$, for $i=1, \ldots, L$, for some $L$. We define the desired function $\phi$ to be

$$
\phi=\sum_{i=1}^{\iota} c_{i} S_{i}
$$




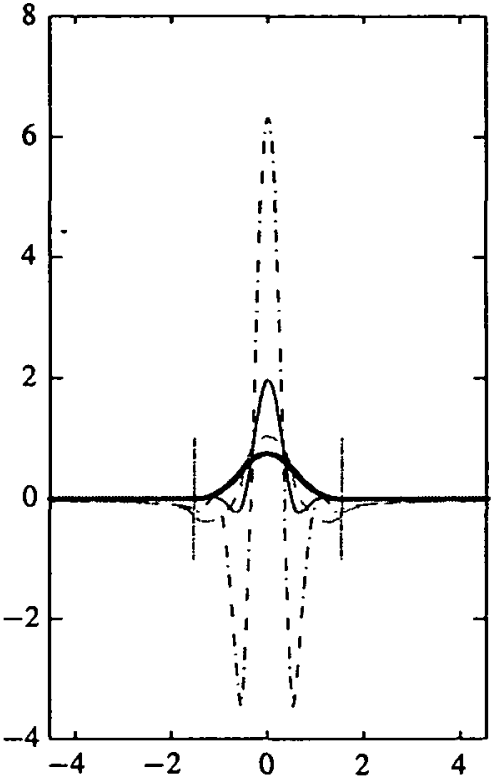

(a)

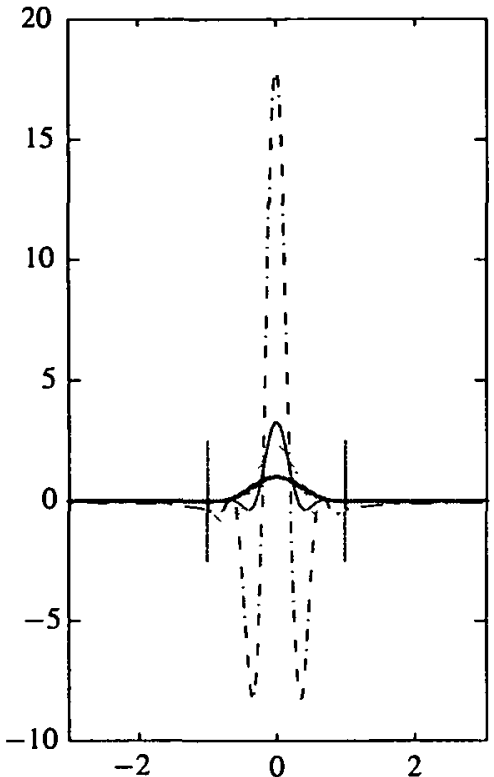

(b)

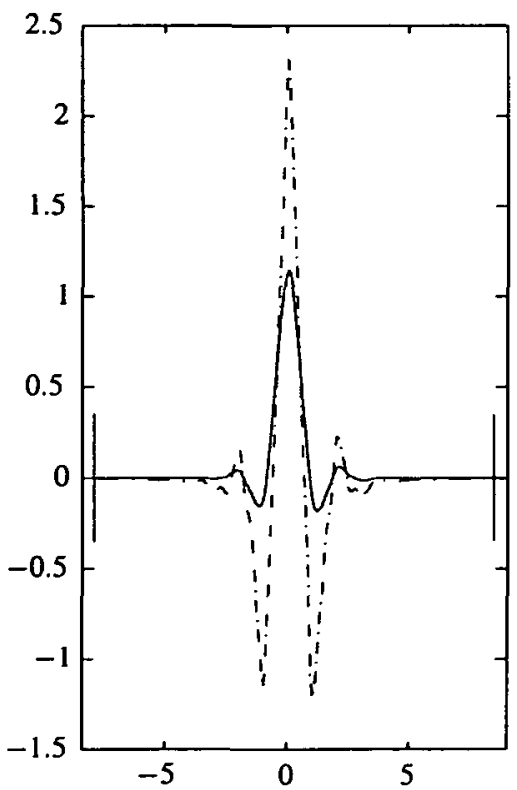

(c)

FIGURE 1. Thick line for $S_{1}$, ' - - ' line for $\Lambda S_{1}$, continuous line for $\phi$ and '- - -' line for $\Lambda \phi$ in both the cases where the $\phi$ used is based on (a) (5.3), (b) (5.4) and (c) a coiflet (coif3). Vertical lines represent the support margins of $\phi$ and $S_{1}$. 
TABLE 1. Coefficients $c_{i}$ in $\phi$ and the spread of the function outside the support after the $\Lambda$ operation in different cases.

\begin{tabular}{|l|r|r|r|r|}
\hline & \multicolumn{4}{|c|}{ Cubic Spline } \\
\hline & $L=1$ & $L=3$ & \multicolumn{1}{c|}{$L=4$} & \multicolumn{1}{c|}{$L=5$} \\
\hline Coeff. & 1.0 & 1.0683 & -0.7219 & 0.4286 \\
$\left(c_{i}\right)$ & & -10.9493 & 16.0089 & -17.2165 \\
& & 17.3653 & -68.5788 & 142.8965 \\
& & & 72.8282 & -376.4922 \\
& & & $\cdot$ & 300.7969 \\
\hline Spread & 19.6 & 3.4363 & \multicolumn{1}{|c|}{2.1845} & 1.6030 \\
\hline & \multicolumn{4}{|c|}{ Polynomial } \\
\hline & $L=1$ & $L=3$ & \multicolumn{1}{|c|}{$L=4$} & $L=5$ \\
\hline Coeff. & 1.23 & 1.3747 & -0.8592 & 0.4564 \\
$\left(c_{i}\right)$ & & -10.4066 & 14.9595 & -15.5829 \\
& & 12.3246 & -47.7579 & 98.5007 \\
& & & 37.9638 & -194.5645 \\
& & & & 116.5074 \\
\hline Spread & 15.8 & 2.9277 & 1.9165 & 1.3928 \\
\hline
\end{tabular}

The constants $c_{t}$ are determined from the following moment conditions:

$$
\int_{\mathbb{R}} x^{2 i} \phi(x) d x=\delta_{i, 0}, \quad i=0, \ldots, L-1 .
$$

In (5.2), we consider only the even order zero moments as the odd order moments are trivially zero for symmetric $\phi$. Now the function $\phi$ is compactly supported, smooth and has higher zero moments up to order $2 L-2$. As examples, we consider $S_{1}$ to be the cubic B-spline (5.3) and a finitely supported polynomial-like function (5.4):

$$
\begin{aligned}
& S_{1}(t)=\left\{\begin{array}{lr}
t^{2} / 2, & -3 / 2 \leq t \leq-1 / 2, \\
-\left(2 t^{2}-6 t+3\right) / 2, & -1 / 2 \leq t \leq 1 / 2, \\
\left(t^{2}-6 t+9\right) / 2, & 1 / 2 \leq t \leq 3 / 2, \\
0, & \text { elsewhere, }
\end{array}\right. \\
& S_{1}(t)= \begin{cases}\left(1-t^{2}\right)^{4}, & -1 \leq t \leq 1, \\
0, & \text { elsewhere. }\end{cases}
\end{aligned}
$$

It may be observed that in (5.4) as an example we have considered the fourth degree in the definition of $S_{1}$ to ensure higher order smoothness for $S_{1}$. In both cases, we consider $S_{i}=S_{1}^{i}$ for $i=1,2, \ldots, L$. In order to show the usefulness of these 
functions for our purpose, we compute the spread of energy of $\Lambda \phi$ outside the support of $\phi$ as a fraction of total energy expressed in percentage terms:

$$
\frac{\|\Lambda \phi\|_{L^{2}(R-S u p p \phi)}}{\|\Lambda \phi\|_{L^{2}(R)}} \times 100
$$

These. are tabulated in Table 1 along with the corresponding $c_{i}$ for $L=1,3,4,5$. From Table 1, it can be concluded that the spread decreases as the number of zero (higher) moments of $\phi$ increases. The spread corresponding to the coiflet wavelet (coif 3 ) is $0.8080 \%$. In view of the above, we conclude that any one of these functions can be used for the purpose of local reconstruction.

\section{Error analysis for the localisation of data}

As we have stated at the beginning of this paper, our objective in the present work is to reconstruct the interior portion or ROI using localised data (that is, the data from that interior region plus some neighbouring data from outside the ROI). The region from which the data is collected and used for interior reconstruction is called the Region-Of-Exposure or ROE. The present section deals with finding the ROE for a given ROI.

The estimate proved in Section 4 is an estimate for determining the approximation error when finite $J$ is in use and which works only for full data. However, we need an estimate of the error within the ROI as the ROE increases. In this section, we find an upper bound for the error incurred due to the localisation of the data to a small ROE in the reconstruction of the image in the ROI. Let $r_{1}$ and $r_{e}$ denote the radii of discs centred at the origin of the $\mathrm{ROI}$ and $\mathrm{ROE}$ respectively. Let $[-a, a]$ be the essential support of the functions $\Lambda \phi$ and $\Lambda \psi$ (note that $\Lambda \eta$ is symmetric whenever the function $\eta$ is). Then the extent of data needed to recover the local region is $r_{e}=r_{t}+a / 2^{J}$, which corresponds to the maximum possible overlap of supports of $\left(R_{\theta} f\right)$ and $(\Lambda \phi)$, with $\|x\| \leq r_{t}$. Analogous consideration holds for the part involving $\psi$. In the following we prove an estimate that relates the error arising due to the use of localised data and the extent of localised data $\left(r_{e}\right)$. From (3.3), we have

$$
\begin{aligned}
f_{\eta_{.} J}(x) & =\int_{0}^{\pi} \int_{\mathbb{R}} R_{\theta} f(t) \Lambda \eta_{J}\left(\left\langle x, u_{\theta}\right\rangle-t\right) d t d \theta \\
& =\int_{0}^{\pi}\left(\int_{|| \mid \leq r_{e}}+\int_{|l|>r_{e}}\right) R_{\theta} f(t) \Lambda \eta_{J}\left(\left\langle x, u_{\theta}\right\rangle-t\right) d t d \theta .
\end{aligned}
$$

Let us define the error function $E_{\eta, J}(x)$ to be

$$
E_{\eta, J}(x)=f_{\eta, J}(x)-\int_{0}^{\pi} \int_{|t| \leq r_{r}} R_{\theta} f(t) \wedge \eta J\left(\left\langle x, u_{\theta}\right\rangle-t\right) d t d \theta
$$


Using the fact that $f$ has compact support in the disc of radius $R,\left|R_{\theta} f(t)\right| \leq 2 R\|f\|_{\infty}$. When $x$ belongs to the circle centred around the origin of radius $r_{i},\left|\left\langle x, u_{\theta}\right\rangle\right| \leq r_{i}$. From the definition of $\Lambda, \Lambda \eta_{J}(t)=2^{2 J} \Lambda \eta\left(2^{J} t\right)$. Using all these relations, we get

$$
\begin{aligned}
\left|E_{\eta, J}(x)\right| & =\left|\int_{0}^{\pi} \int_{R>|t|>r_{e}} R_{\theta} f(t) \Lambda \eta_{J}\left(\left\langle x, u_{\theta}\right\rangle-t\right) d t d \theta\right| \\
& \leq 2 \pi R\|f\|_{\infty} \int_{0}^{\pi} \int_{R>|t|>r_{e}}\left|\Lambda \eta_{J}\left(\left\langle x, u_{\theta}\right\rangle-t\right)\right| d t d \theta \\
& =2 \pi R\|f\|_{\infty} \int_{0}^{\pi} \int_{R>\left|t-\left\langle x, u_{\theta}\right)\right|>r_{e}}\left|\Lambda \eta_{J}(t)\right| d t d \theta \\
& \leq 2 \pi R 2^{J}\|f\|_{\infty} \int_{0}^{\pi} \int_{2^{J}\left(R+r_{s}\right)>|t|>2^{J}\left(r_{e}-r_{i}\right)}|\Lambda \eta(t)| d t d \theta \\
& \leq 2 \pi^{2} R 2^{J}\|f\|_{\infty} \int_{|| \mid>2^{J}\left(r_{e}-r_{i}\right)}|\Lambda \eta(t)| d t .
\end{aligned}
$$

In (6.2), the fourth step follows from the third step due to the following reasons. The relations $\left|\left\langle x, u_{\theta}\right\rangle\right| \leq r_{i}$ and $R>\left|t-\left\langle x, u_{\theta}\right\rangle\right|>r_{e}$ imply $R+r_{i}>|t|>r_{e}-r_{t}$. Since $\left\{t: R>\left|t-\left\langle x, u_{\theta}\right\rangle\right|>r_{e}\right\} \subset\left\{t: R+r_{i}>|t|>r_{e}-r_{i}\right\}$, the inequality symbol follows in the fourth step. Using the relation $\Lambda \eta_{J}=2^{2 J} \Lambda \eta\left(2^{J} t\right)$ and replacing $2^{J} t$ with $t$, we get the fourth step. Finally, with $\phi$ in the place of $\eta$, we have

$$
\frac{\left\|E_{\phi, J}\right\|_{L^{\infty}(\mathrm{ROI})}}{R\|f\|_{L^{\infty}\left(\mathbb{R}^{2}\right)}} \leq 2 \pi^{2} 2^{J} \int_{|l|>2^{J}\left(r_{e}-r_{1}\right)}|\Lambda \phi(t)| d t .
$$

Observe that the right-hand side of the above inequality is independent of $f$. From the above inequality, it may be concluded that at a given $J$, as $r_{e}$ increases, the right-hand side of (6.3) decreases. The decay of the right-hand side of (6.3) against the increase in $\left(r_{e}-r_{1}\right)$ is shown in Figure 2 using the functions $\phi$ constructed based on (5.3), (5.4) at $L=3$ (5.1) and the coiflet (coif3). From the graphs, which are independent of $r_{t}$ and the test image $f$, it may be concluded that when $r_{e}=r_{i}+a / 2^{J}$ for $a>4.5$ (in the first case), $a>3$ (in the second case) and $a>10$ (in the third case), the error arising out of localisation of data to the interior region becomes exceedingly small. There is yet another type of error due to the discretisation of data. It is, however, quite standard to take care of it by sampling the data using Nyquist sampling rates (as dictated by the essential band limit of $\phi$ ).

Although from the above error analysis we get information about the radius of the region of exposure, in the simulation part, we start with a $\phi$ with support $[-a, a]$ (say). Then we assume that $\Lambda \phi$ has essential support $[-\nu, \nu]$ with increasing values of $v$ starting with $a$. We see how the error and quality of the reconstructed local image change in the course of simulation. 


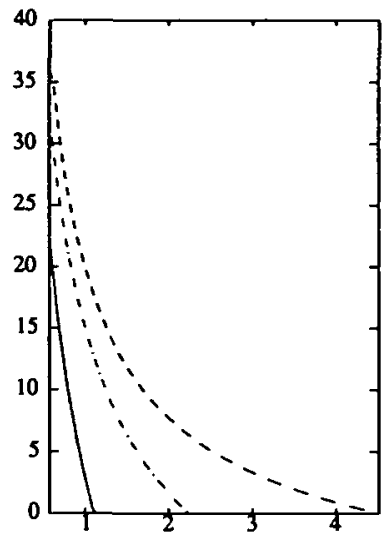

(a)

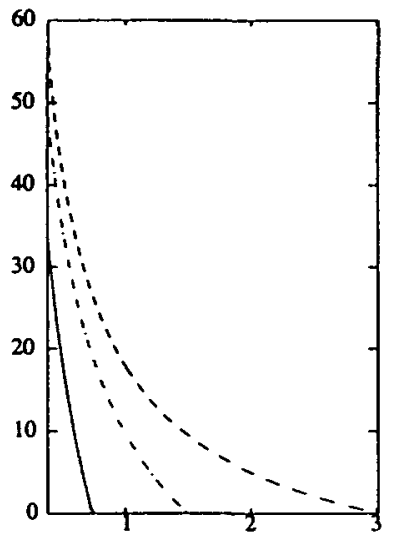

(b)

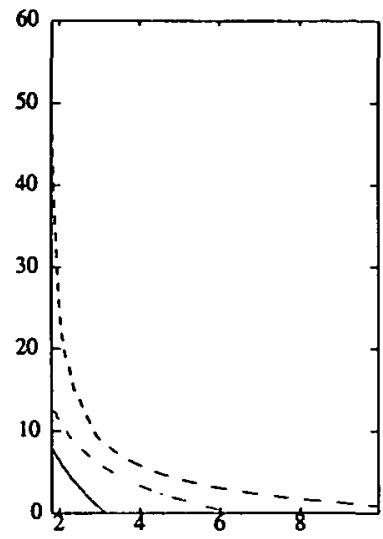

(c)

FIGURE 2. Plot between $\left(r_{e}-r_{i}\right)$ and the right-hand side of (6.3). The function $\phi$ used is based on (a) (5.3), (b) (5.4), and (c) a coiflet (coif3). In all three cases, we use a continuous line for $J=3, a^{\prime}-\cdot-$ ' line for $J=2$, and a ' -- ' line for $J=1$.

\section{Some comparisons}

Although the algorithm presented in this section is not wavelet based, nevertheless, a comparison of it with the algorithm promoted by Faridani et al. in [8-10] seems worthwhile as both the methods in some way use approximation identities for certain functions. The $\Lambda_{F}$ operator

$$
\begin{aligned}
\Lambda_{F} f(x) & =(-\Delta)^{1 / 2} f(x) \\
& =-\frac{1}{4 \pi} \Delta \Re^{\#} \Re f(x)=-\frac{1}{4 \pi} \int_{0}^{2 \pi} \partial^{2}\left(R_{\theta} f\right)\left(\left\langle x, u_{\theta}\right\rangle\right) d \theta
\end{aligned}
$$

studied by Faridani et al. is local and is aimed at capturing a certain high frequency part of $f$. In (7.1), $\Delta$ represents the Laplace operator. According to the authors of [8-10], in practical applications of $\Lambda$-tomography, they do not compute $\Lambda_{F} f$, but rather attempt to reconstruct $\Lambda_{F}\left(\eta_{\epsilon} \star f\right)(x)[13,17]$ for some approximation identity $\eta_{\epsilon}(x)=\epsilon^{-2} \eta(x / \epsilon), \epsilon>0$, where $\eta$ is an integrable function with total integral one. Now for a good choice of $\epsilon, \Lambda_{F} f(x)$ is computed via

$$
\begin{aligned}
\Lambda_{F} f(x) \approx \Lambda_{F}\left(\eta_{\epsilon} \star f\right)(x) & =\left[\epsilon^{-1}\left(\Lambda_{F} \eta\right)_{\epsilon} \star f\right](x) \\
& =\left(e_{\epsilon} \star f\right)(x)=R^{\#}\left(v_{\epsilon} \star \Re f\right)
\end{aligned}
$$

In the above, $e_{\epsilon}=\epsilon^{-1}\left(\Lambda_{F} \eta\right)_{\epsilon}$ and $v_{\epsilon}=\Lambda \Re e_{\epsilon}$. Finally, $e_{\epsilon} \star f$ is computed via the back projection method. In the end, the image that one gets is not an approximation of the original image, but of the high frequency part of $f$. To include the missing low 
frequency part of $\Lambda_{F} f$, another operator $L f$ given by

$$
L f:=\Lambda_{F} f+\mu \Lambda_{F}^{-1} f
$$

has been studied and analysed in [9] and [10]. The operator $\Lambda_{F}^{-1}$ included in $L$ represents the inverse of the operator $\Lambda_{F}$ and (see [13]) is given by

$$
\Lambda_{F}^{-1} f(x)=\frac{1}{4 \pi} \Re^{\#} \Re f(x)=\frac{1}{4 \pi} \int_{0}^{2 \pi}\left(R_{\theta} f\right)\left(\left\langle x, u_{\theta}\right\rangle\right) d \theta .
$$

Here again the function $L f$ is a local function which does not represent $f$ but a related function [10]. The authors of [9] and [10] have studied and demonstrated the effects of $\Lambda_{F}$ and $\Lambda_{F}^{-1}$ and have considered the coefficient $\mu$ by trial and error in their experiments. Although we also introduce a parameter $\lambda$ with a view to enhancing the reconstructed image, we observe that the role of $\lambda$ is marginal when we consider larger values of $J$. We discuss this aspect in the section dealing with simulation. But most importantly, the inclusion of $\lambda$ (or the $\psi$-part) is not mandatory in our procedure for reconstruction. Hence no question of comparison between the roles of $\lambda$ in (3.4) and $\mu$ in (7.3) arises.

The method established by A. K. Louis [11] uses the concept of approximate identity for the purpose of regularisation. In [11], the method involves solving the equation $R^{*} v=e$ for a mollifier (approximate identity) $e$ with $\int e(x) d x=1$ by $v=(2 \pi)^{-1} \Lambda R e$. The method is then given as

$$
f_{\gamma}(x)=R f \star D^{\gamma, x} v,
$$

where $D^{\gamma, x} v(\theta, t)=\gamma^{-2} v\left(\theta,\left(t-\left\langle x, u_{\theta}\right\rangle\right) / \gamma\right)$.

Although the method proposed in the present work uses the properties of approximation identities of certain classes of functions, it differs in its objective from the other algorithms using the same concept. The basic intention in the present work is the reconstruction of the original local image as accurately as possible using local data by using a CBP like procedure.

The approximate computation of $\Lambda_{F} f$ and the estimation of associated approximation errors have been the subject of study by Rieder $e t$ al. in [17]. The basic objective of the authors in [17] is to investigate the properties of a compactly supported $v$, where $v=\Lambda \Re e$ in (7.2) and $\Lambda_{F} f \approx f \star e_{f}$. An error estimate is derived by taking a zero moment condition for $v$ for even integers (that is, $\int s^{2 k} v(s) d s=0$, for $k=0,1$ ).

In (4.4), $\Lambda \phi$ plays the same role as $v$ in (7.2). Although, for symmetric $\phi, \Lambda \phi$ satisfies the same zero mean and zero moment conditions as $v$, it lacks compact support. Hence the estimate given in [17] is not applicable in the present context.

Basically, the method given here involves one level and the methods involving wavelet decompositions use a hierarchy of levels $[5,7,15]$. In addition the waveletbased decompositions involve translations which also add to the computations. 


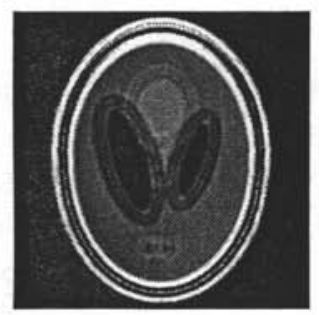

(a)

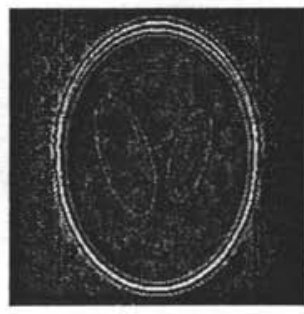

(b)

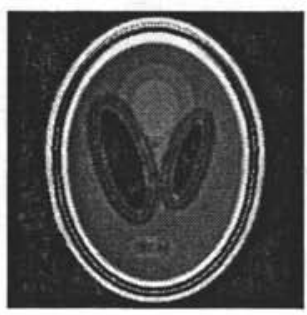

(c)

FIGURE 3. (a) $f_{\phi, 0}$, (b) $5 f_{\psi, 0}$ and (c) $f_{\phi, 0}+f_{\psi, 0}$.

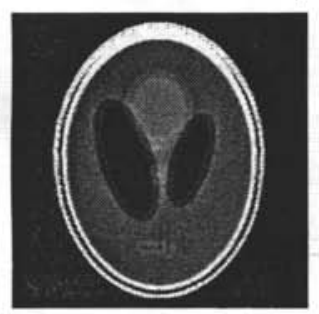

(a)

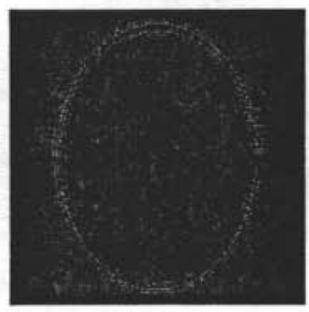

(b)

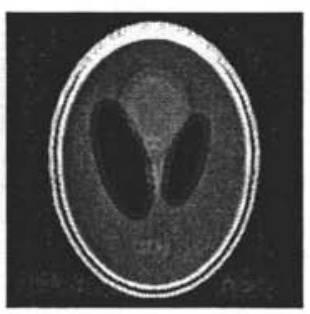

(c)

FIGURE 4. (a) $f_{\phi, 1}$, (b) $5 f_{\psi, 1}$ and (c) $f_{\phi, 1}+f_{\psi, 1}$.

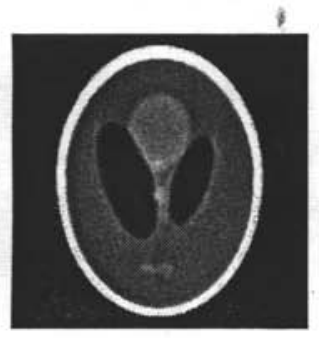

(a)

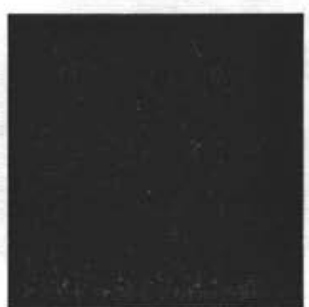

(b)

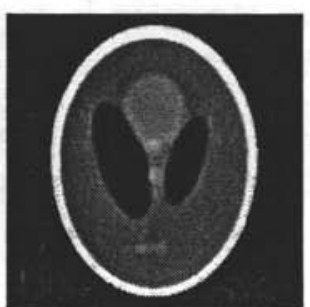

(c)

FIGURE 5. (a) $f_{\phi, 2}$, (b) $5 f_{\psi, 2}$ and (c) $f_{\phi, 2}+f_{\psi, 2}$.

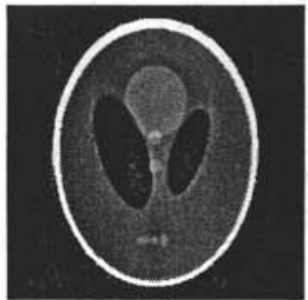

(a)

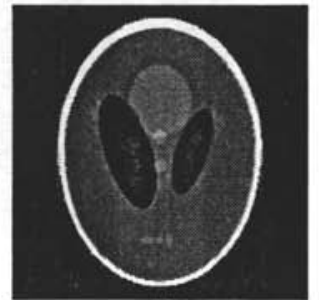

(b)

FIGURE 6. (a) $f_{\phi, s}+f_{\psi, 5}$ and (b) original test image. 


\section{Results of simulation}

As stated earlier, a wide variety of $\phi$ and $\psi$ can be used in simulation. Since the inclusion of $\psi$ is not at all compulsory in (3.4), one can carry out the simulations using the $\phi$ functions alone constructed in Section 5. However, we present in the following the results obtained using the coiflet pair $\phi$ and $\psi$ and study the usefulness of the $\psi$-part when the $\psi$ considered is orthogonal to $\phi$.

We have carried out computations using the coiflet ('coif3') wavelet and scaling functions for different $J$. Reconstructions of the Shepp-Logan head phantom have been executed on $256 \times 256$ pixel grids using 256 projections collected at 256 uniformly spaced angles over $[0, \pi)$ and the reconstructed images are shown in Figures 3-6. It may be observed that these figures are displayed by discarding the portion lying outside the outer ellipse to make the changes occurring in the main part more visible. In all of Figures 3-5, the wavelet part is shown with $\lambda=5$ to emphasise the nature of the contribution of $f_{\psi, J}$ to $f$. We have computed the $L^{\infty}, L^{2}$ errors in $\%$ terms using the formula

$$
L^{p} \text { error }(\text { in } \%)=\frac{\left\|f-f_{r}\right\|_{p}}{\|f\|_{p}} \times 100, \quad p=2, \infty,
$$

for the cases $\tilde{\phi}=\phi$ as well as $\tilde{\phi}=\phi+\psi$ (that is, $\lambda=1$ ). Here, $f_{r}$ stands for the reconstructed image. The errors computed are shown in Table 2. It may be observed from Table 2 that the $\psi$ part does not change the errors significantly for $J \geq 3$ and the $L^{\infty}$ error comes below $1 \%$ for $J \geq 6$.

TABLE 2. $L^{p}, p=2, \infty$, errors in $\%$ with $\phi, \phi+\psi$ in place of $\tilde{\phi}$ at different values of $J$.

\begin{tabular}{|c|c|c|c|c|}
\hline$J$ & $L^{\infty}(\%): \phi$ & $L^{\infty}(\%): \phi+\psi$ & $L^{2}(\%): \phi$ & $L^{2}(\%): \phi+\psi$ \\
\hline 0 & 221.36 & 221.23 & 79.67 & 75.13 \\
\hline 1 & 232.34 & 230.53 & 44.84 & 44.14 \\
\hline 2 & 131.09 & 130.71 & 12.71 & 12.64 \\
\hline 3 & 47.187 & 47.179 & 1.618 & 1.617 \\
\hline 4 & 12.945 & 12.945 & 0.124 & 0.124 \\
\hline 5 & 3.3125 & 3.3125 & 0.008 & 0.008 \\
\hline 6 & 0.8330 & 0.8330 & $5.197 \times 10^{-4}$ & $5.197 \times 10^{-4}$ \\
\hline 7 & 0.2085 & 0.2085 & $3.2591 \times 10^{-5}$ & $3.2591 \times 10^{-5}$ \\
\hline 8 & 0.0522 & 0.0522 & $2.0386 \times 10^{-6}$ & $2.0386 \times 10^{-6}$ \\
\hline 9 & 0.0130 & 0.0130 & $1.2744 \times 10^{-7}$ & $1.2744 \times 10^{-7}$ \\
\hline 10 & 0.0033 & 0.0033 & $7.9655 \times 10^{-9}$ & $7.9655 \times 10^{-9}$ \\
\hline
\end{tabular}

When the 'coif3' wavelet is in use for local reconstruction, the excess (outside the ROI) data to be used amounts to just 6 pixels only for $J=0$ (that is, the radius of the 
TABLE 3. $L^{p}, p=2, \infty$, errors in $\%$ with constant and zero extensions of data outside the ROE at different values of $J$.

\begin{tabular}{|c|c|c|c|c|c|}
\hline$J$ & $\begin{array}{c}\text { Nonlocal } \\
\text { data(pixels) }\end{array}$ & $\begin{array}{c}L^{\infty}(\%) \\
\text { Const. Ext. }\end{array}$ & $\begin{array}{c}L^{\infty}(\%) \\
\text { Zero Ext. }\end{array}$ & $\begin{array}{c}L^{2}(\%) \\
\text { Const. Ext. }\end{array}$ & $\begin{array}{c}L^{2}(\%) \\
\text { Zero Ext. }\end{array}$ \\
\hline 0 & $6+0$ & 86.556 & 85.831 & 46.540 & 105.811 \\
\hline 0 & $6+6$ & 85.309 & 68.755 & 43.572 & 57.268 \\
\hline 0 & $6+10$ & 84.295 & 61.537 & 41.153 & 41.902 \\
\hline 1 & $3+0$ & 64.612 & 83.466 & 31.547 & 127.103 \\
\hline 1 & $3+6$ & 64.115 & 65.883 & 30.458 & 58.285 \\
\hline 1 & $3+10$ & 63.169 & 60.349 & 28.067 & 36.694 \\
\hline 2 & $2+0$ & 45.349 & 78.145 & 25.419 & 137.145 \\
\hline 2 & $2+6$ & 45.279 & 56.222 & 25.601 & 59.6701 \\
\hline 2 & $2+10$ & 44.304 & 48.417 & 23.265 & 35.408 \\
\hline 3 & $1+0$ & 28.813 & 72.293 & 22.511 & 155.977 \\
\hline 3 & $1+10$ & 28.379 & 35.945 & 21.785 & 38.136 \\
\hline 4 & $1+0$ & 21.564 & 71.229 & 22.183 & 155.229 \\
\hline 4 & $1+6$ & 21.769 & 42.266 & 23.509 & 65.4887 \\
\hline 4 & $1+10$ & 20.751 & 31.169 & 21.455 & 37.785 \\
\hline 5 & $1+0$ & 21.092 & 71.438 & 22.154 & 155.089 \\
\hline 5 & $1+6$ & 21.211 & 42.112 & 23.480 & 65.441 \\
\hline 5 & $1+10$ & 19.911 & 30.954 & 21.427 & 37.752 \\
\hline 6 & $1+4$ & 21.404 & 49.668 & 23.909 & 86.526 \\
\hline 6 & $1+6$ & 21.139 & 42.189 & 23.478 & 65.435 \\
\hline 6 & $1+8$ & 20.593 & 36.086 & 22.642 & 49.664 \\
\hline 6 & $1+10$ & 19.839 & 31.013 & 21.412 & 37.740 \\
\hline 6 & $1+15$ & 18.192 & 21.282 & 18.495 & 18.854 \\
\hline 6 & $1+20$ & 16.552 & 14.739 & 15.311 & 9.029 \\
\hline 6 & $1+25$ & 16.602 & 9.779 & 14.831 & 3.930 \\
\hline
\end{tabular}

$\mathrm{ROE}$ is the radius of the $\mathrm{ROI}+9$ for $J=0$ ). Lower numbers for the ROE can possibly be achieved using several other suitable pairs given in [4]. In all the computations, the ROI is the central $1 / 4$ portion of the full image (that is, radius $=32$ pixels). We have made computations for local reconstructions at different values of $J$. To avoid the artifacts caused due to the truncation of data to the ROE, we have considered a constant extension of the data outside the ROE as expressed through the equation

$$
\left(R_{\theta} f\right)(t)=\left(R_{\theta} f\right)\left(r_{e}\right) \text { for all }|t| \geq r_{e} \text { and } \theta
$$

Otherwise, the sharp cut off (discontinuities) introduced into the data function may have an adverse impact on the quality of the outputs due to Gibb's phenomenon. However, in computations, we have estimated the errors on the ROI both with the constant as well as with zero extension of data outside the ROE and the recordings 


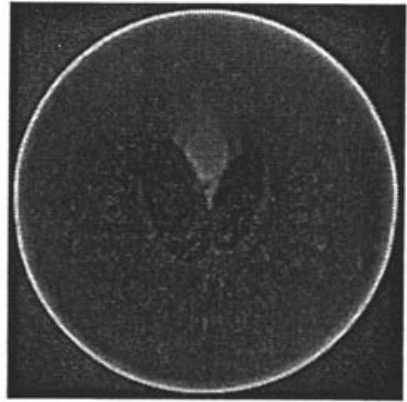

(a)

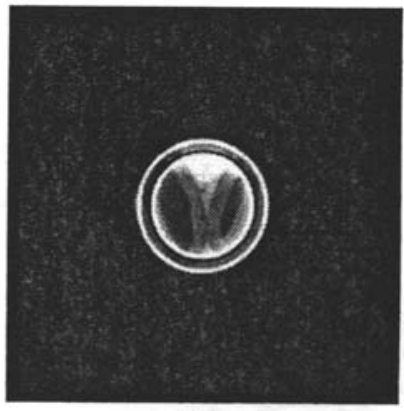

(b)

FIGURE 7. Reconstruction on the ROI of radius 32 pixels at $J=0$, that is, $f_{\phi, 0}$ with (a) constant extension, (b) zero extension of data outside the ROE.

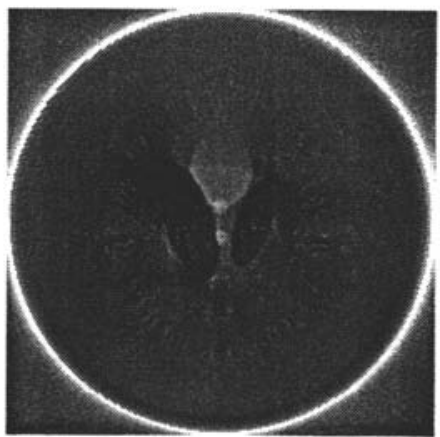

(a)

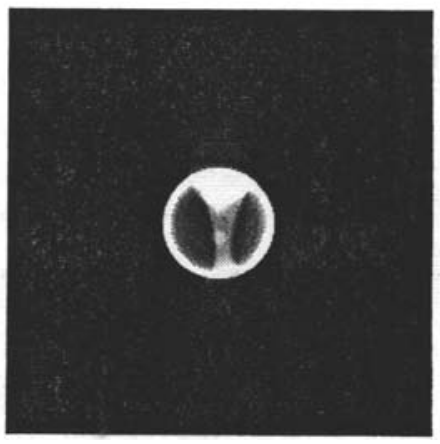

(b)

FIGURE 8. Reconstruction on the ROI of radius 32 pixels at $J=3$, that is, $f_{\phi, 3}$ with (a) constant extension, (b) zero extension of data outside the ROE.

are shown in Table 3 . In the second column of Table 3, we have shown the nonlocal data in ' $p+q$ ' form, where ' $p$ ' denotes the overlap of supports of $\left(R_{\theta} f\right)$ and the ramp function outside the ROI (that is, $a / 2^{J}$ in terms of pixels-as stated in the last paragraph of Section 5) and ' $q$ ' stands for the excess data that we supply to reduce the errors. From Table 3 it may be observed that the $L^{\infty}$ error (in $\%$ terms) shows a very slow decline in value when we increase the ROE after $J=4$.

As was pointed out in [12], the interior reconstruction procedures give a constant shift in the absolute value of the density function to be found. We have observed that a removal of a constant bias with value -0.14 from the reconstructed image at $J=4$ and $\mathrm{ROE}=11$ pixels results in a fall in $L^{\infty}$ error to $3 \%$ from nearly $20 \%$. The reconstructed ROI images shown in Figures 7-9 suggest that with an excess margin of 11 pixels one can have good local images. It may be observed that the $\psi$-part in 


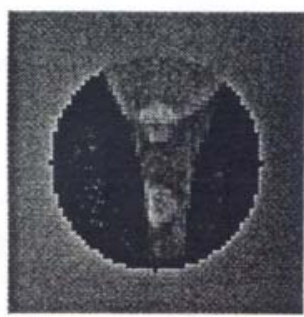

(a)

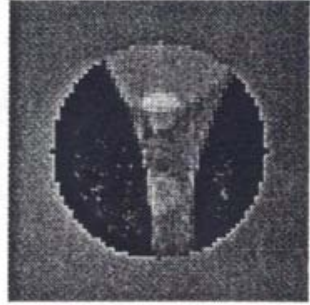

(b)

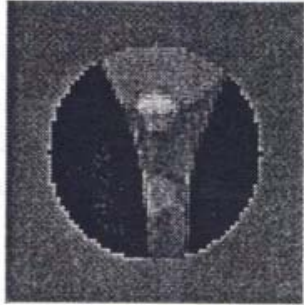

(c)

FIGURE 9. Reconstruction on the ROI of radius 32 pixels at $J=4$, (a) before removing constant bias and (b) after removing constant bias (c) original local image obtained via the CBP procedure.

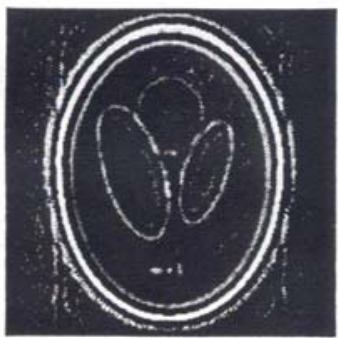

(a)

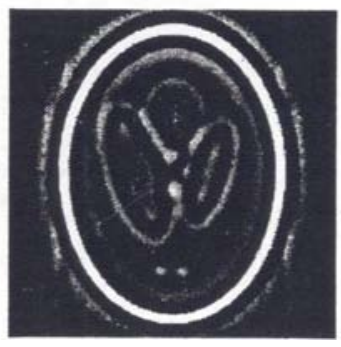

(b)

FIGURE 10. $5 f_{\psi, J}$ at (a) $J=-1$ and (b) $J=-2$.

the coiflet case gives negligible contribution to $f$. We have observed the errors for different $J$ 's and $\lambda$ 's (that is, $\tilde{\phi}=\phi+\lambda \psi$ ) and our conclusion is that for positive $J, f_{\psi, J}$ 's role is negligible. However, for negative $J, f_{\psi, J}$ captures edge information as shown in Figure 10. Hence the inclusion of the $\psi$-part with negative $j$ into the representation, that is, with $\tilde{\phi}=\phi_{j}+\lambda \psi_{-j}$ for some appropriate $j$ and $\lambda$, may enhance the edges present in the images. Since the choice of $j$ and $\lambda$ is case dependent for image enhancement problems, we will not go into the details of computational work in this regard. It is theoretically justified that the $\phi$ functions constructed in Section 5 work well for local reconstructions. We have observed similar results, as presented in these sections, using the $\phi$ constructed in Section 5. To avoid presenting too many results, we omit them here.

\section{Conclusion}

The algorithm proposed in the present work uses space and frequency localisation properties of certain functions having zero moments. These functions form an ap- 
proximation identity and remain essentially compactly supported after ramp filtering. The algorithm retains the structure of the filtered back projection (FBP) procedure and admits faster and standard ways of implementation using a relatively small amount of data for recovering local regions.

\section{Acknowledgements}

The authors thank the referee for his/her suggestions. The first author is grateful to the Council of Scientific and Industrial Research (CSIR), India for its financial support (Grant No. FNO:9/92 (164)/98-EMR-1).

\section{References}

[1] R. A. Adams, Sobolev spaces (Academic Press, New York, 1975).

[2] A. Brandt, J. Mann, M.!Brodski and M. Galun, "A fast and accurate multilevel inversion of the Radon transform", SIAM J. Appl. Math. 60 (2000) 437-462.

[3] C. K. Chui, An introduction to wavelets (Academic Press, New York, 1992).

[4] I. D. Cohen and J. C. Feauveau, "Biorthogonal bases of compactly supported wavelets", Comm. Pure. Appl. Math. 45 (1992) 485-560.

[5] P. C Das and Ch. S. Sastry, "Region-of-interest tomography using a composite Fourier-wavelet algorithm", Num. Funct. Anal. 23 (2002) 757-777.

[6] I. Daubechies, Ten lectures on wavelets, CBMS-NSF Series in Appl. Math. 61 (SIAM, Philadelphia, 1992).

[7] J. Destefano and T. Olson, "Wavelet localization of Radon transform", IEEE Trans. Signal Process. 42 (1994) 2055-2067.

[8] A. Faridani, E. L. Ritman and U. T. Smith, "Local tomography", SIAM J. Appl. Math. 52 (1992) 459-484.

[9] A. Faridani, E. L. Ritman and U. T. Smith, “Local tomography Il”, SIAM J. Appl. Math. 57 (1997) 1095-1127.

[10] A. Faridani, K. A. Buglione, P. Huabsomboon, O. D. Iancu and J. McGrath, "Introduction to local tomography", Contemp. Math. 278 (2000) 29-47.

[11] A. K Louis, "Approximate inverse for linear and some nonlinear problems", Inverse Problems 12 (1996) 175-190.

[12] A. K. Louis and A. Rieder, "Incomplete data problems in X-ray computerized tomography II: Truncated projections and region-of-interest tomography", Numer. Math. 56 (1989) 371-383.

[13] W. R. Madych, "Tomography, approximate reconstruction,and continuous wavelet transforms", Appl. Comput. Harmon. Anal. 7 (1999) 54-100.

[14] F. Natterer, The mathematics of computerised tomography (Wiley, New York, 1986).

[15] F. R. Rashid-Farrokhi, K. J. R. Liu, C. A. Berenstein and O. Walnut, "Wavelet based multi resolution local tomography", IEEE Trans. Image Process. 6 (1997) 1412-1430.

[16] A. Rieder, "Principles of reconstruction filter design in 2D-computerized tomography", Contemp. Math. 278 (2000) 201-226.

[17] A. Rieder, R. Dietz and T. Schuster, "Approximate inverse meets local tomography", Math. Meth. Appl. Sci. 23 (2000) 1373-1387.

[18] B. Sahiner and A. E. Yagle, "Region-of-interest tomography using exponential radial sampling", IEEE Trans. Image Process. 4 (1995) 1120-1127. 